\title{
New Solutions in Online Sheet Thickness Measurements in Incremental Sheet Forming
}

\author{
Imre Paniti
}

\begin{abstract}
This work discusses an approved analytical framework of Single Point Incremental Forming (SPIF) of sheet metals, which is capable of modelling the state of stress in the small localised deformation zone in case of corners, flat and rotationally symmetric surfaces. The discussion focuses on the investigation of the sheet thickness prediction in the shell element used in the framework. Novel solutions are introduced in terms of on-line sheet thickness measurement and an adaptive control algorithm in SPIF. Results were experimentally verified for $0.5 \mathrm{~mm}$ thick Al 1050 sheets with variable wall angle geometry. Furthermore, a new device for two sided incremental sheet forming is introduced as a recently patented solution in Incremental Sheet Forming (EP2505279).
\end{abstract}

\section{Nomenclature}

$F \quad$ Reaction force

$F_{x} \quad$ X-component of the reaction force

$F_{y} \quad$ y-component of the reaction force

$F_{z} \quad$ Z-component of the reaction force

$r \quad$ Radial coordinate

$r_{\text {tool }}$ Radius of the forming tool

$r_{1} \quad$ Radius of curvature of meridian at the shell element (equal to $r_{\text {tool }}$ )

$r_{2} \quad$ Radius of the element normal where it cuts the $\mathrm{z}$-axis

$T \quad$ Measurement time

$t \quad$ Thickness of the sheet

$t_{0} \quad$ Initial thickness of the sheet

$U \quad$ Hall voltage

I. Paniti (凶)

Institute for Computer Science and Control, Hungarian Academy of Sciences,

Budapest Kende u. 13-17, 1111, Hungary

e-mail: imre.paniti@sztaki.mta.hu 


\section{Greek Symbols}

$\alpha$ Defining the angle in the meridional plane at the shell element

$\beta \quad$ Wall angle

$\epsilon_{\theta}$ Circumferential strain

$\epsilon_{\varphi}$ Meridional strain

$\mu_{\theta}$ Circumferential component of the coefficient of friction

$\mu_{\varphi}$ Meridional component of the coefficient of friction

$\sigma_{m}$ Mean stress

$\sigma_{t} \quad$ Thickness stress

$\sigma_{Y}$ Yield stress

$\sigma_{\theta} \quad$ Circumferential stress

$\sigma_{\varphi}$ Meridional stress

\section{Introduction}

The study and the improvement of Incremental Sheet Forming (ISF) have been of considerable interest during the last couple of decades. The interest in these scientific investigations is because this process is flexible (the geometry of the formed part can be changed by the control of the machine without changing the tool) and enables higher formability as compared to conventional sheet forming technologies like stamping or deep-drawing. ISF is considered to be used only for small series and Rapid Prototypes, but the applicability of this process has a wide range in the fields of the automotive industry, aircraft industry, architectural engineering and medical aids manufacturing.

The basic idea of ISF is similar to metal spinning and goes back to a patent granted in 1967 by Leszak [12]. As opposed to metal spinning, where the sheet metal is formed into an axial symmetric object by being turned on a lathe and being pressed continually with a tool, in ISF the sheet is clamped down with a frame and formed with a flat or spherical head forming tool. In contrast to conventional plastic deformation processes the final shape of the part is not determined by specific dies, rather by the three-dimensional movement of a forming tool, for which an appropriate tool path has to be established, based on the part geometry. This movement can be carried out by a CNC milling machine or by an industrial robot. ISF can be divided into two main groups [10] depending on the number of contact points between sheet, forming tool and die (see Fig. 1).

In Single Point Incremental Forming (SPIF) the first contour of the part is supported by a backing plate without any specific die. In case of Two Point Incremental Forming (TPIF) a full or partial die is applied with a downward movement of the clamping frame [17]. 


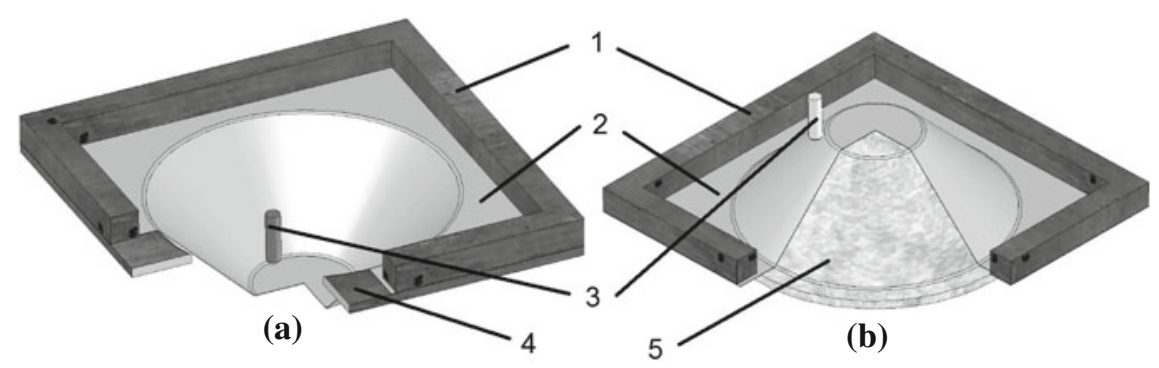

Fig. 1 a SPIF and $\mathbf{b}$ TPIF where 1 clamping frame, 2 sheet, 3 forming tool, 4 backing plate, 5 full or partial die

\section{Analytical Background of SPIF}

The analytical model introduced by Silva et al. [20] is based on membrane analysis with bi-directional in-plane contact friction forces to study the influence of major process parameters and their interaction.

This model uses the following simplifications:

- thickness, meridional and circumferential stresses assumed to be principal stresses;

- bending moments are neglected;

- axial symmetry is assumed;

- the material is simplified to be isotropic and rigid-perfectly plastic;

- the resultant friction stress which acts at the tool-sheet contact interface is assumed to consist of a meridional and a circumferential in-plane component.

Silva et al. [20] examined the smear-marks intervention between the forming tool and the surface of the sheet and concluded that the instantaneous small plastic zone of rotational symmetric SPIF can be approximated by a local shell element CDEF, illustrated in Fig. 2.

Figure 3 shows the acting stresses of the shell element in a schematic section view cut by an axial meridional plane. Figure 3 a shows the top; and $b$ the detail view. The

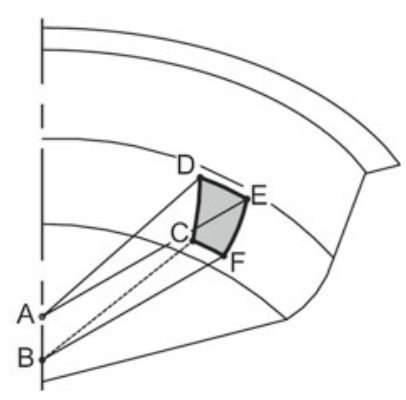

Fig. 2 Schematic perspective drawing of the shell element [20] 
(a)

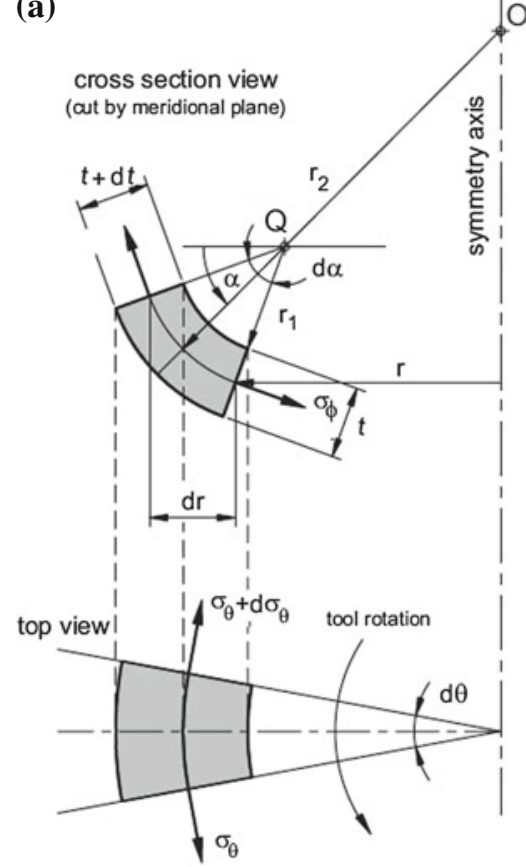

(b)

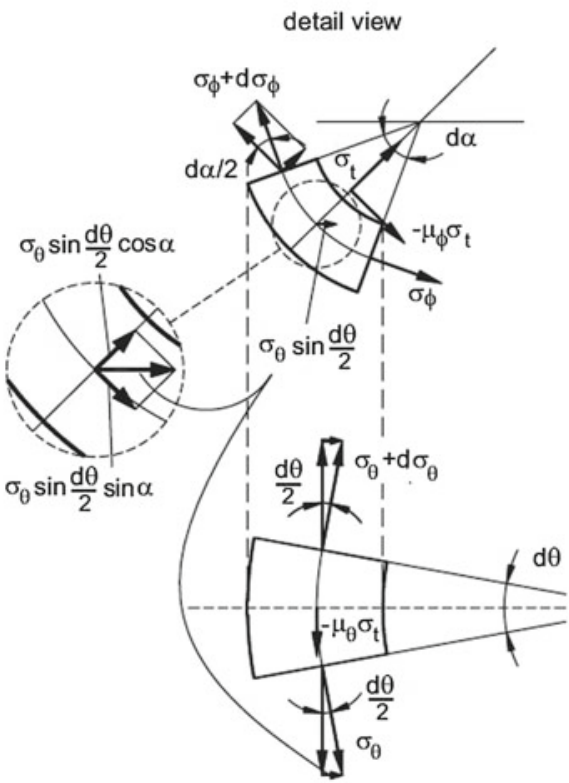

Fig. 3 Acting stresses of the shell element in a schematic section view cut by an axial meridional plane, with a top view, and b detail view [20]

normal of the local shell element cuts the $\mathrm{z}$-axis at the point $\mathrm{O}$ and $r_{2}$ is defined as the corresponding radius. Point $\mathrm{Q}$ represents the centre of curvature on the normal and $r_{1}$ is the radius of curvature of the local shell element along the meridian direction [20].

By resolving the force equilibrium along the meridional, thickness and circumferential direction the following equations can be written (see [20]).

Along the meridional direction:

$$
\begin{array}{r}
\left(\sigma_{\varphi}+d \sigma_{\varphi}\right)(r+d r) d \theta(t+d t)-\sigma_{\varphi} r d \theta t+\mu_{\varphi} \sigma_{t} r d \theta r_{1} d \alpha \\
-\sigma_{\theta} \frac{d \theta}{2} r_{1} d \alpha t \sin \alpha-\left(\sigma_{\theta}+d \sigma_{\theta}\right) \frac{d \theta}{2} r_{1} d \alpha t \sin \alpha=0
\end{array}
$$

Along the thickness direction:

$$
\begin{array}{r}
\sigma_{t} r d \theta r_{1} d \alpha+\sigma_{\varphi} r d \theta t \sin \frac{d \alpha}{2}+\left(\sigma_{\varphi}+d \sigma_{\varphi}\right)(r+d r) d \theta(t+d t) \sin \frac{d \alpha}{2} \\
+\sigma_{\theta} r_{1} d \alpha t \sin \frac{d \theta}{2} \cos \alpha+\left(\sigma_{\theta}+d \sigma_{\theta}\right) r_{1} d \alpha t \sin \frac{d \theta}{2} \cos \alpha=0
\end{array}
$$


Along the circumferential direction:

$$
\sigma_{\theta} r_{1} d \alpha\left(t+\frac{d t}{2}\right)-\mu_{\theta} \sigma_{t} r_{1} d \alpha\left(r+\frac{d r}{2}\right) d \theta-\left(\sigma_{\theta}+d \sigma_{\theta}\right) r_{1} d \alpha\left(t+\frac{d t}{2}\right)=0
$$

Furthermore the model assumes that the flat and rotationally symmetric surfaces are formed under plane-strain conditions, $d \epsilon_{\theta}=0$, and corners are formed under equal biaxial stretching, $d \epsilon_{\varphi}=d \epsilon_{\theta}>0$. Under these conditions, considering the Tresca yield criterion-which states that $\sigma_{1}-\sigma_{3}$ should be constant and equal to the flow stress of the sheet - the distribution of stresses in the small localised plastic zones can be obtained from Eqs. (1), (2) and (3) after neglecting higher order terms and taking into account geometrical simplifications [21].

In the case of flat and rotationally symmetric surfaces the mean stress can be written in the following simplified form:

$$
\sigma_{m}=\frac{\sigma_{Y}}{2}\left(\frac{r_{t o o l}-t}{r_{\text {tool }}+t}\right)
$$

For corners the mean stress can be written in the following simplified form:

$$
\sigma_{m}=\frac{2 \sigma_{Y}}{3}\left(\frac{r_{\text {tool }}-t}{r_{\text {tool }}+2 t}\right)
$$

\section{Sheet Thinning in SPIF}

In 2009, Silva et al. [21] compared the analytical model described in the previous section with FEM simulations and experiments, and results showed good qualitative agreement. However, the thickness of the sheet is approximately estimated by means of the sine-law, originated from the spinning process [5];

$$
t=t_{0} \sin \left(90^{\circ}-\beta\right) \text {. }
$$

The parameter $t_{0}$ is the initial thickness of the sheet and $\beta$ is the wall angle (defining the angle between the un-deformed and the deformed sheet). In 2004, Young and Jeswiet [25] analysed radial profiles of truncated cones with various wall angles formed by SPIF. Results showed that sheet thinning in SPIF does not always follow the sine-law; the formula can only be used in case of low strains and in some cases the estimation of (6) is higher than in reality.

In 2010, Bambach [6] summarised different versions of the sine-law, e.g. where the thickness change is calculated as a function of the area ratio of the un-deformed and deformed triangular or quadrilateral mesh elements, and proposed a geometrical model of the kinematics of ISF for the prediction of membrane strains and sheet thickness. This model gives more accurate results than the sine-law, as it corrects the 
sine-law in curved areas and reproduces it on flat surfaces. However, the model has some limitations too.

Bambach emphasised that "due to the fact that material behaviour and friction are not included in the model it will not give accurate results in cases in which the sheet thickness is affected by the material behaviour in reality, e.g. for large wall angles where necking can occur or in situations dominated by friction" [6, p. 1571].

\section{Sheet Thickness Measurements in SPIF}

Due to the above mentioned deficiencies in the prediction of the sheet thickness in SPIF it is important to apply an on-line measurement method as a supplement. Based on the on-line thickness measurement an adaptive control could be applied to delay or even avoid fracture.

Some stereo vision systems are capable of measuring on-line the surface strains, so thickness strains can be estimated, but experimental investigations showed that only initial stages can be analysed with one paint layer [9]. The use of a paint layer with a pattern is necessary in order to trace the movements of material points under SPIF.

The experimental investigations of Van Bael et al. [24] and Aerens et al. [2] showed that the steady-state value of the axial reaction force during the SPIF process is proportional to the wall angle and to the resulting wall thickness. These force measurements in ISF are usually carried out using a three-component piezoelectric dynamometer, but other solutions like the application of piezo-resistive or strain gauged sensor system is also suitable. It is worth mentioning that forming force estimation can be carried out also from servomotor torque measurements taking into account the mechanical equilibrium of the machine tool structure used for the ISF process [18].

In 2006, Ambrogio et al. [3] claimed that the punch force trend is suitable as a "spy variable" of approaching material failure in the case of excessive sheet thinning. However, the previously mentioned measurement solutions can only provide indirect approximations as opposed to direct sheet thickness measurements.

In 2010, Dejardin et al. [7] also emphasised that the on-line monitoring of sheet thinning is important for the industrialisation of SPIF and for the process optimisation. They introduced a new solution for in-process thickness measurements based on the integration of an ultrasonic thickness measurement device into a forming tool. Dejardin et al. reported a measurement accuracy mean equal to $\pm 0.01 \mathrm{~mm}$ with proper repeatability [7]. However, one disadvantage of this approach is that the immersion transducer requires a propagation media with a specific viscosity. An accurate measurement could only be obtained with a $70 \%$ water ratio. The other disadvantage of this solution is that the set-up can only be used in machines which are able to follow the normal vector defined by the Tool Centre Point (TCP) and by the contact point of the sheet. The orientation change along the tool path is indispensable, because sheet thinning occurs in the small plastic zone defined in [20]. 


\section{Novel On-Line Sheet Thickness Measurement Solutions in SPIF}

In 2011, Paniti and Paróczi [15] introduced a different on-line solution for in-process thickness measurements which can be applied in SPIF. The authors analysed the possibility of using a Hall-effect sensor integrated into a flat incremental forming tool. The investigations in FEM modelling and simulation showed good results in the design phase regarding the measurement possibilities, but the flat forming tool design of the first prototype limited the orientation of the tool.

By keeping the measurement principle, an inverse set-up allows to measure the thickness in the small plastic zone. For the inverse set-up the forming tool suggestions of Leszak [12] — which have similarities to a ballpoint pen-gave a good starting point. The forming tool in this case is a bearing ball with free rotation possibility. To measure the thickness in SPIF with the same solution suggested in [15] a Hall-effect sensor and a magnet have to be placed on the other side of the sheet. This inverse set-up makes self-adjusting measurement possible, because the magnet follows the bearing ball along the programmed tool path and the orientation of the set-up is adjusted according to the thickness of the sheet in the small plastic zone.

\subsection{Calibration of the Thickness Measurement Device}

The measurement set-up consists of three main elements:

- a steel target ball (like a G100 CARBON type bearing ball with $10 \mathrm{~mm}$ diameter as used in our experiments);

- a linear-output Hall transducer (like an AD22151 type Hall sensor IC from Analog Devices in bipolar configuration [1] as used in our experiments);

- and a permanent magnet (like an AlNiCo permanent magnet with $10 \mathrm{~mm}$ length and $10 \mathrm{~mm}$ diameter as used in our experiments) that provides a magnetic field.

The magnetic flux density varies monotonically as a function of displacement, but the Hall voltage will be significantly nonlinear with respect to position. In this case the magnetic flux density is inversely proportional to the sheet thinning. For an accurate sheet thickness measurement it is important to define a calibration curve with discrete data acquisition. Figures 4 and 5 shows the calibration device with a micrometre screw as the carrier tool for the bearing ball.

Calibration measurements were carried out with 3 series (300 measurement points in each series) using a Motion Control and Data Acquisition PCI card [14] for the A/D conversion. Every $50 \mathrm{~ms}$ a 16-point moving average was applied as a low-pass filter. It is important to choose an adequate distance between the Hall sensor and the magnet, because a too high value could interfere with the motion of the device (see curve "magnet-sensor distance: $3 \mathrm{~mm}$ " in Fig. 6), while on the other hand a too small value could cause an overflow in the measurement (see curve "magnet-sensor distance: 1 mm" in Fig. 6). 


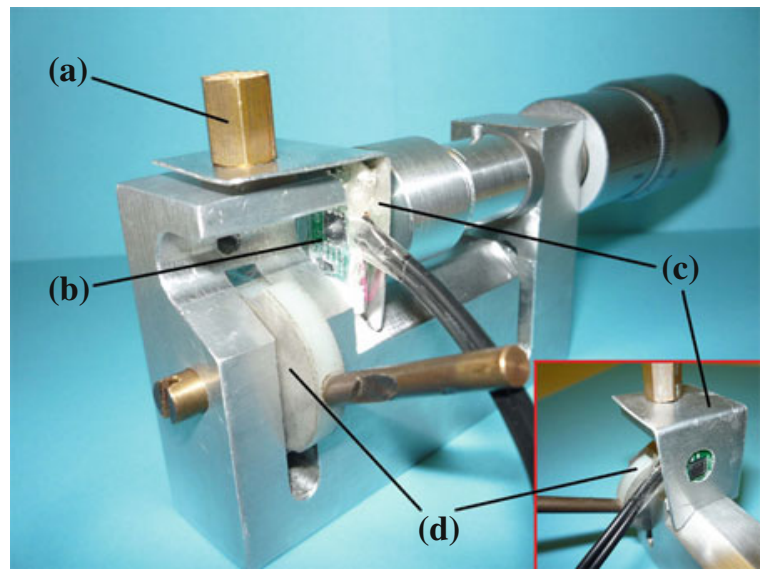

Fig. 4 Calibration set-up of the Hall sensor based thickness measurement device with a sheet mounting part, $\mathbf{b}$ Hall sensor, $\mathbf{c}$ bent $\mathrm{Al}$ sheet, $\mathbf{d}$ magnet mounting part

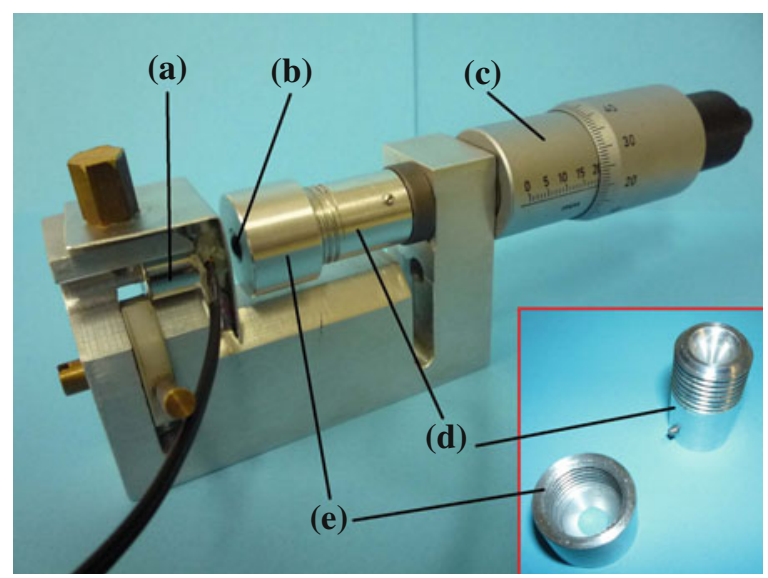

Fig. 5 Calibration set-up of the Hall sensor based thickness measurement device with a magnet, $\mathbf{b}$ bearing ball, $\mathbf{c}$ micrometre screw, $\mathbf{d} \mathrm{Al}$ housing of the bearing ball, e cap of the Al housing

Figure 7 shows the calibration curve up to $1 \mathrm{~mm}$ with an adequate magnet-sensor spacer glued between the AlNiCo magnet and the Hall sensor.

A 6th order polynomial regression in this case gives the following equation with a correlation value close to 1 .

$$
U=26813 t^{6}-12207 t^{5}+19028 t^{4}-12168 t^{3}+2586.1 t^{2}-244.32 t+3848.9
$$




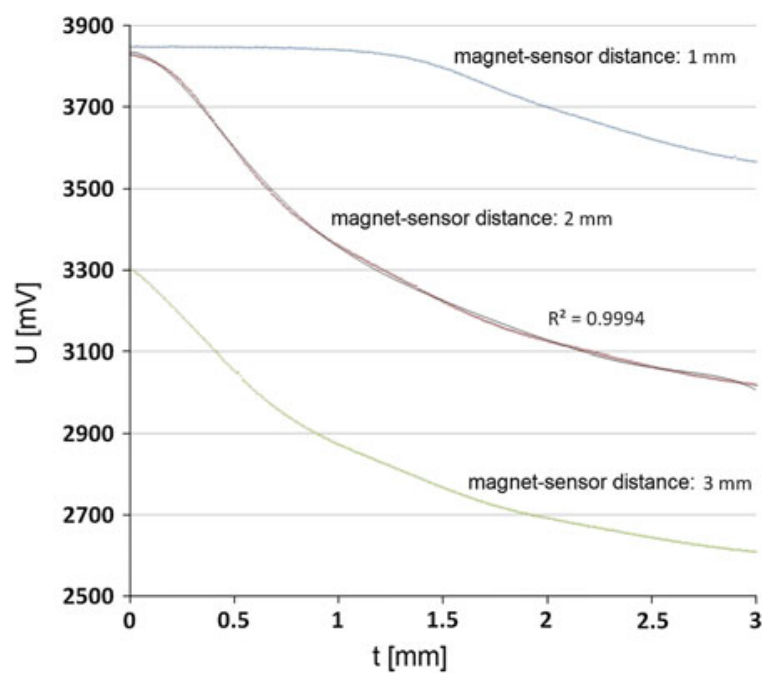

Fig. 6 Calibration curves with different magnet-sensor distances

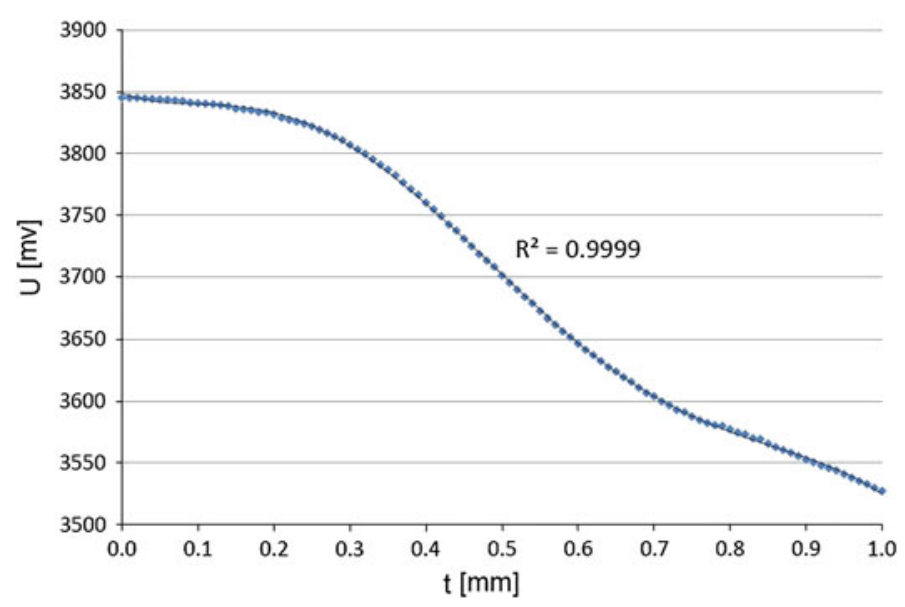

Fig. 7 Calibration curve of the prototype

\subsection{Proof of Concept with Adaptive Control Algorithm}

In 2004, Hirt et al. [10] summarised the influence of the SPIF process parameters on the final product and on each other. They concluded that sheet formability decreases by increasing the step depth.

In 2006, the experimental investigations of Attanasio et al. [4] showed the importance of using a tool path that has a variable step depth, because a lower step depth increases accuracy_but on the other hand it increases the process time too, thus leading to inefficient production. 


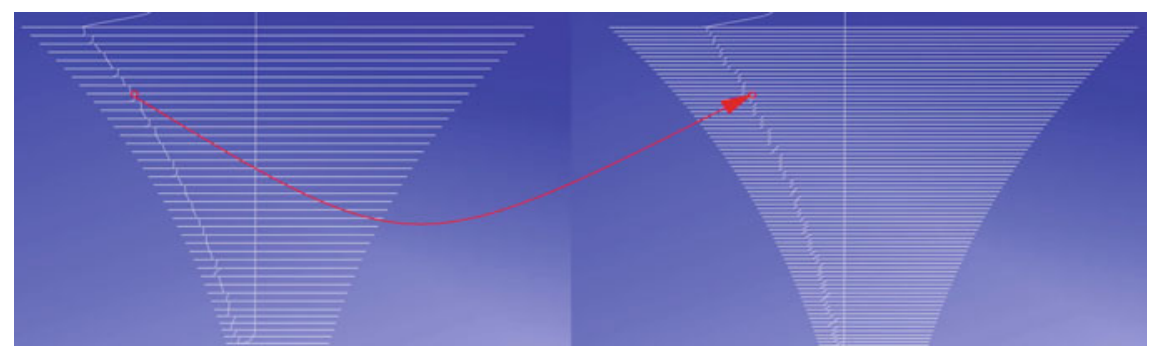

Fig. 8 Illustration of tool path change from 1 to $0.5 \mathrm{~mm}$ step depth

A possible solution could be the application of an on-line thickness measurement method with a simple adaptive control algorithm, which changes the step depth to a lower value. To guarantee the same final shape and depth of the product as with the initial step depth, several tool paths based on the same geometry but with different step depths are needed [16].

Figure 8 shows the two tool paths applied in the experiments with constant 1 and $0.5 \mathrm{~mm}$ step depths and shows a possible tool path change as example.

Hussain and Gao [11] demonstrated that the number of experiments required to determine the forming limit of a sheet can be reduced by using a part geometry with variable wall angle. For this reason and because of the sheet thinning observations discussed in Sect. 3 the experiments were carried out with a variable wall angle $\beta$, by using the part geometry presented in Fig. 9.

Force measurements with a JR3 type Multi-axis Force and Torque sensor are carried out simultaneously to prove the applicability of the monitoring concept by

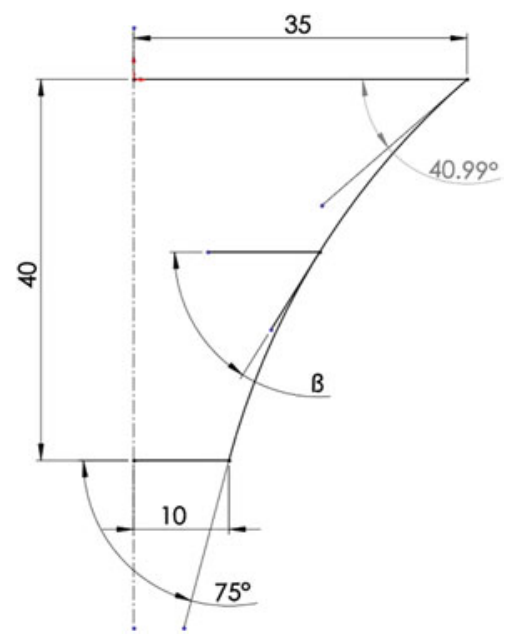

Fig. 9 Part geometry with variable wall angle 


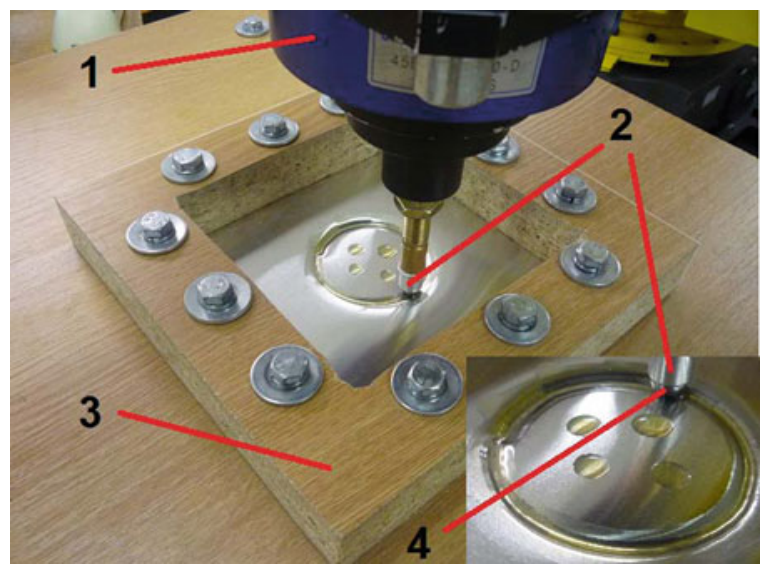

Fig. 10 SPIF set-up with 1 Force cell, 2 bearing ball (forming tool) holder, 3 clamping frame and 4 bearing ball as forming tool

Ambrogio et al. [3] on the part geometry. The force cell was mounted between the tool holder and the flange of a FANUC S430iF industrial robot.

In order to measure the sheet thickness with the Hall sensor based solution, but without disturbing materials in the close environment of the sensor, the clamping frame, the backing plate and the forming tool (bearing ball) holder in the set-up need to be non-ferromagnetic. Figure 10 shows the realisation of the previous mentioned criteria.

Machine oil was applied as lubricant on the surface of the sheet to minimise friction. Figure 11 shows the forming and the measurement set-up from below.

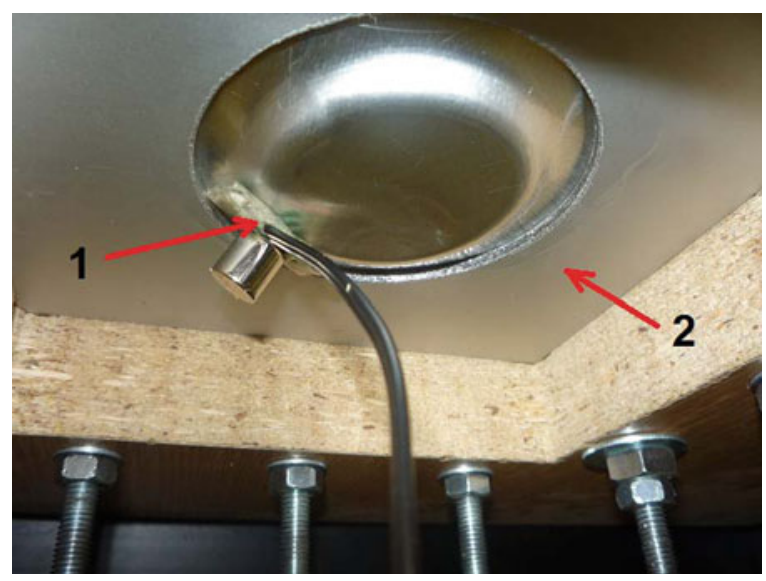

Fig. 11 SPIF set-up from below with 1 Hall sensor based thickness measurement device and 2 backing plate 


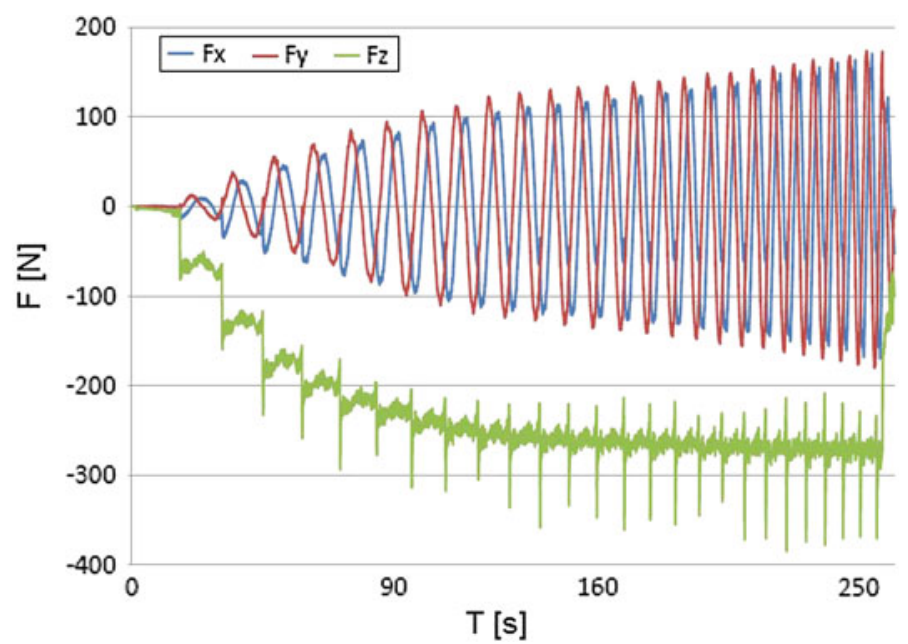

Fig. 12 Reaction force curves with sharp drop-down of the z-component (preliminary test)

Preliminary SPIF experiments were carried out with $0.5,0.7$ and $0.8 \mathrm{~mm}$ thick $\mathrm{Al}$ 1050 sheets and some experimental investigations were reported with $0.6 \mathrm{~mm}$ thick sheets in [23]. Despite the high wall angles in the part geometry, only those sheets with $0.5 \mathrm{~mm}$ initial thickness showed fracture in all cases. For this reason the target of the experiments was limited to the Al 1050 sheets with $0.5 \mathrm{~mm}$ initial thickness.

Due to the relative low magnetic density of the applied AlNiCo magnet the forming speed had to be limited to $9 \mathrm{~mm} / \mathrm{s}$ to obtain reliable measurement results. Figure 12 shows a preliminary test with reaction force components measured by the Force cell till fracture.

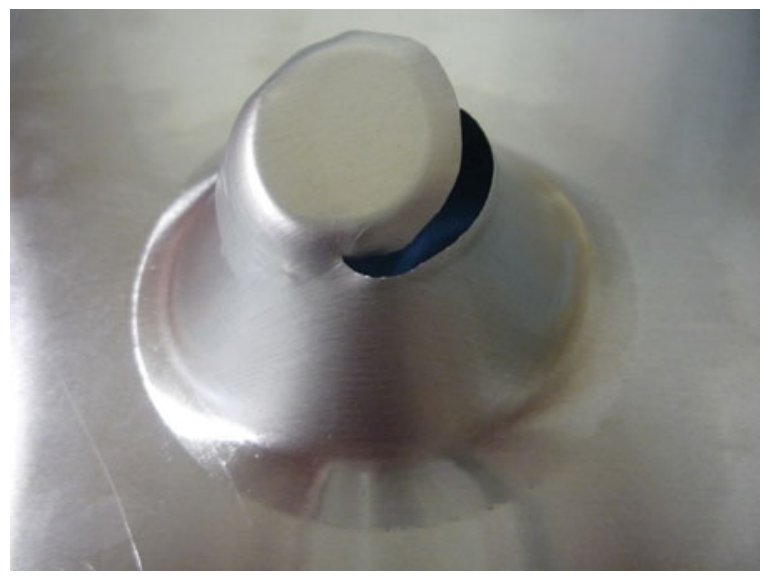

Fig. 13 Photo of the formed part with circumferential crack propagation (preliminary test) 


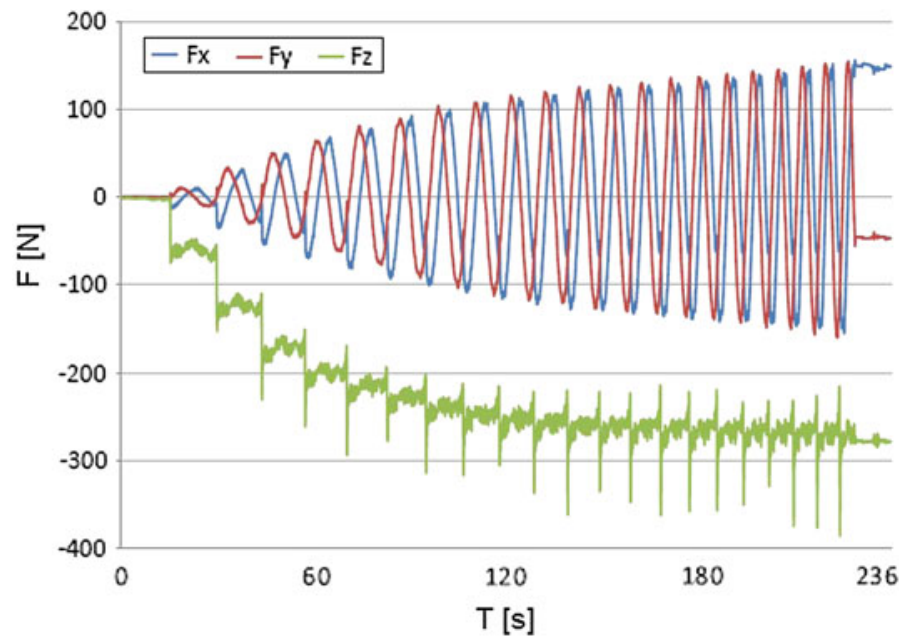

Fig. 14 Reaction force curves without significant change at the critical value ( 1 test)

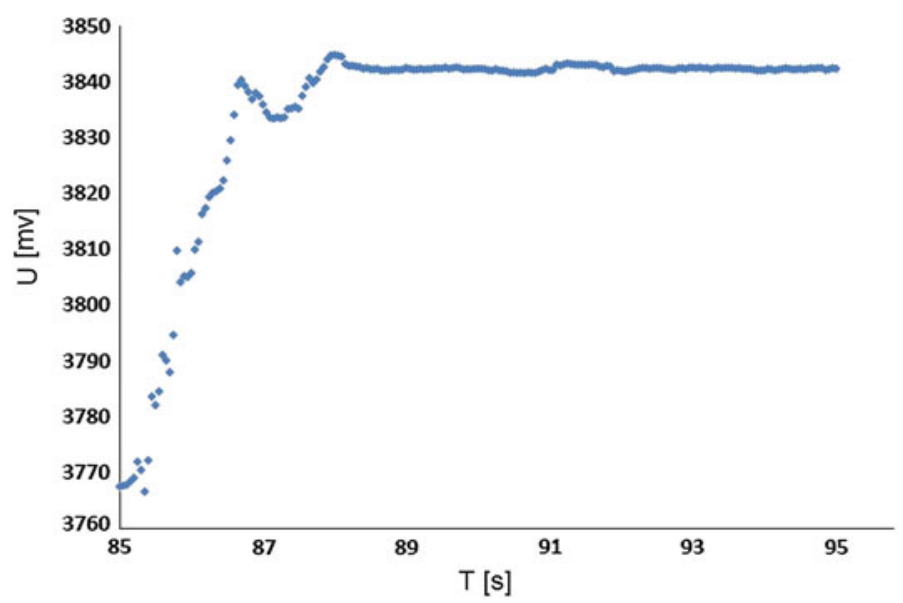

Fig. 15 Measurement results of the Hall sensor around the critical value ( 1 test) 


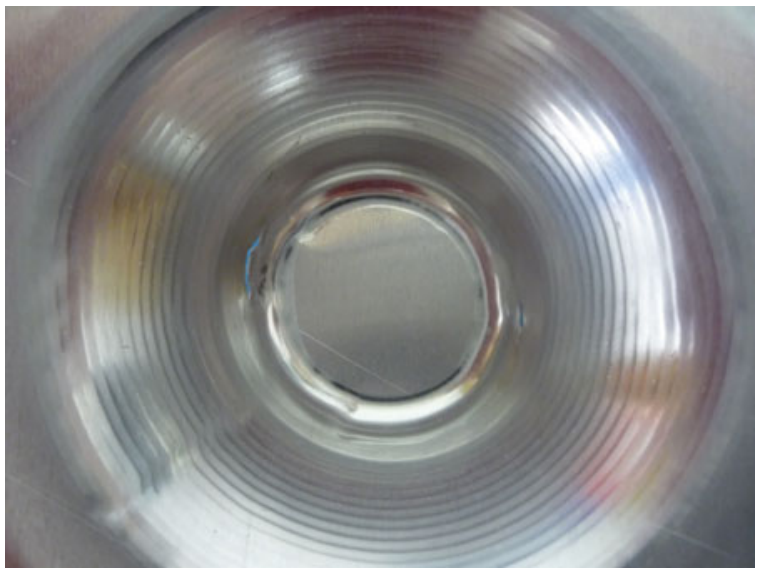

Fig. 16 Photo of the formed part with changed tool path and crack propagation ( 1 test)

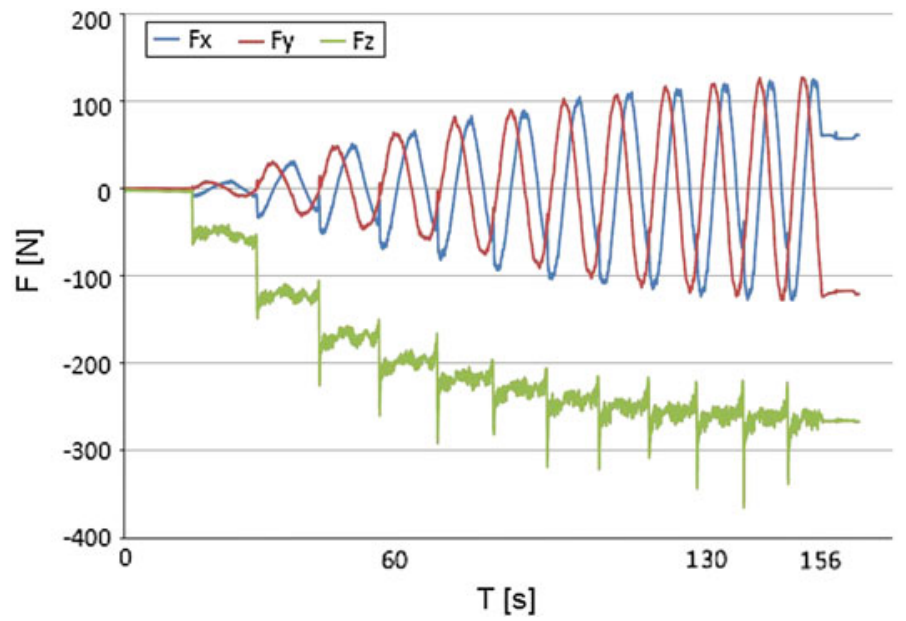

Fig. 17 Reaction force curves without significant change at the critical value (2 test) 


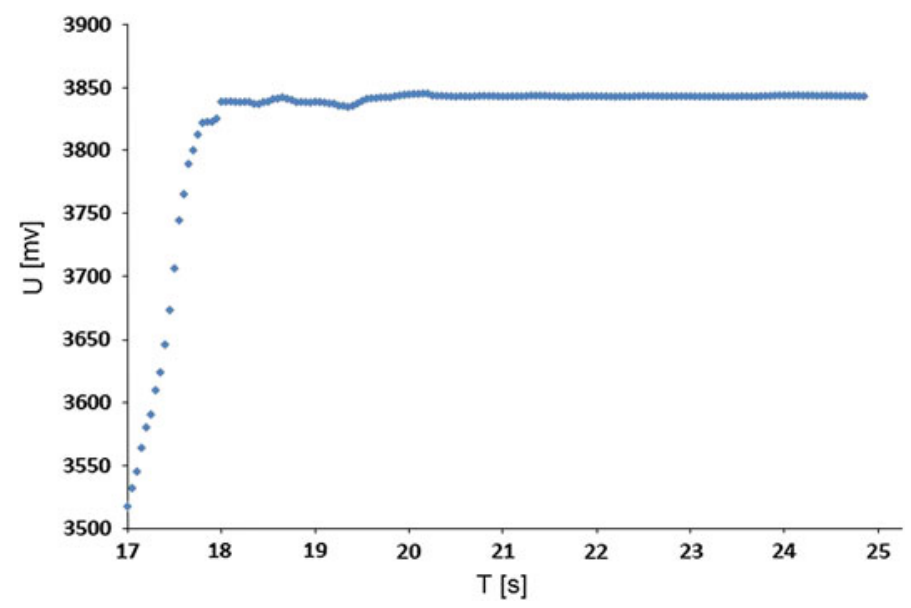

Fig. 18 Measurement results of the Hall sensor around the critical value (2 test)

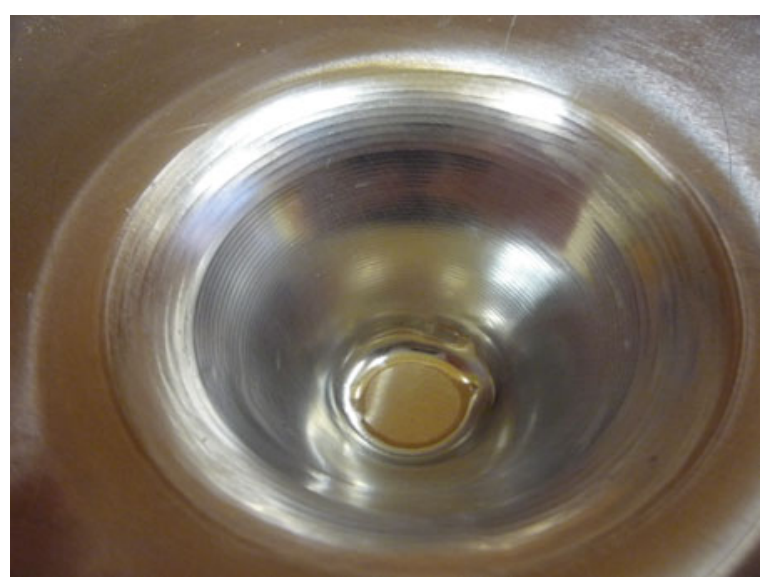

Fig. 19 Photo of the formed part with local thinning at the beginning of the forming (2 test) 


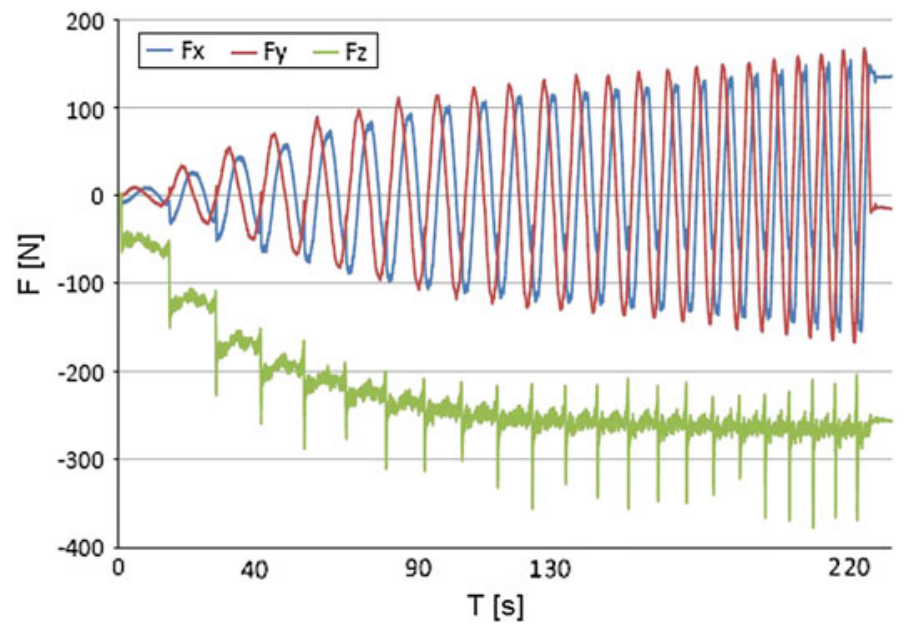

Fig. 20 Reaction force curves without significant change at the critical value (3 test)

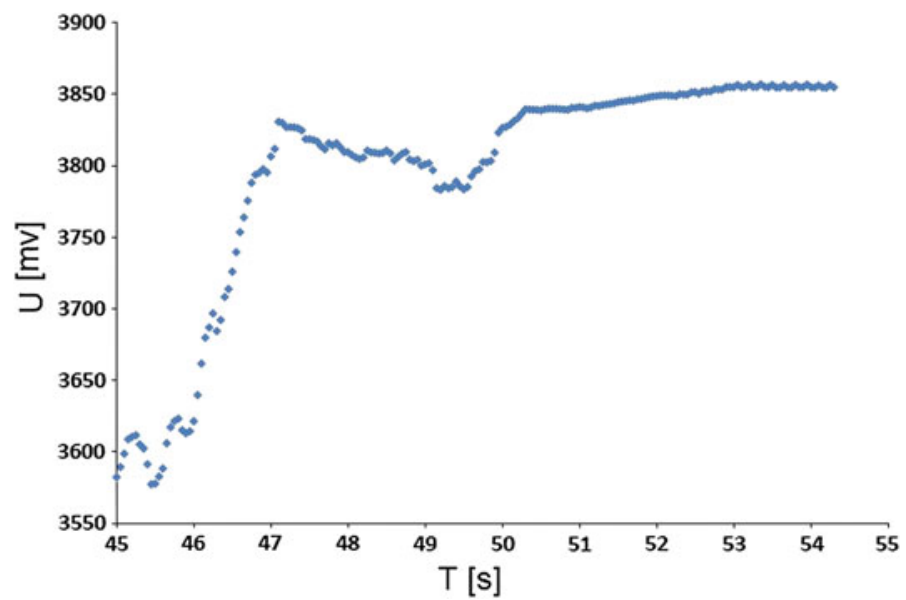

Fig. 21 Measurement results of the Hall sensor around the critical value with a "stabilised thickness area" (3 test) 


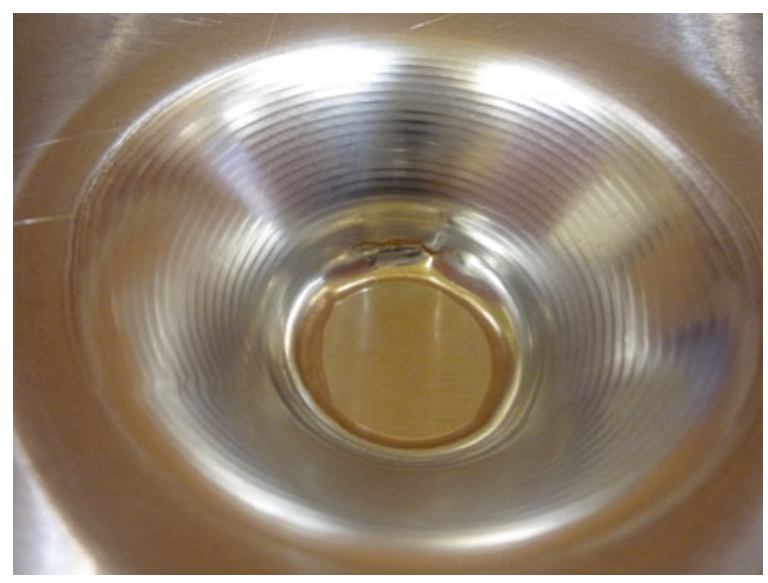

Fig. 22 Photo of the formed part with changed tool path and crack propagation (3 test)

A clear drop in the $\mathrm{z}$-component $(\mathrm{Fz})$ could be observed at the time of the fracture. The result of the forming on the part is shown by Fig. 13.

Hall voltage around $3840 \mathrm{mV}$ was measured at the fracture point. This corresponds to a thickness of $0.11 \mathrm{~mm} \pm 0.01 \mathrm{~mm}$. The thickness measurement was carried out after the forming to determine the critical value at the fracture point. Figures 14, 17 and 20 shows the reaction force components (for the same part) up to the critical value obtained by the Hall sensor based thickness measurement device before the tool path change.

The current prototype size of the Hall sensor based thickness measurement device and the backing plate did not allow the measurements to be made from the beginning. Results (Figs. 15, 18, 21) show the last 150-200 measurement points around the critical value for the same part, where the forming was stopped.

Figure 16 shows the formed part with 1 and $0.5 \mathrm{~mm}$ step depths. Crack occurred after the tool path change.

Figure 19 shows that a local thinning occurred with a critical value at the beginning of the forming. The process was stopped according to the algorithm and a tool path change was started. The tool path with $0.5 \mathrm{~mm}$ step depth formed the part almost completely.

Figure 21 shows a "stabilised thickness area" like in [25] without continuous thinning. The critical value is reached twice in this case. Figure 22 shows the formed part of the third test with changed tool path and crack propagation.

This thickness measurement solution is limited to non-ferromagnetic materials, but if the conditions are adequate (technical properties are selected properly and the device is secured against flipping to the lower flat zone of the sheet with e.g. a solution such as that reported in [22]) mean measurement accuracy equal to \pm 0.01 $\mathrm{mm}$ with proper repeatability can be reached. 


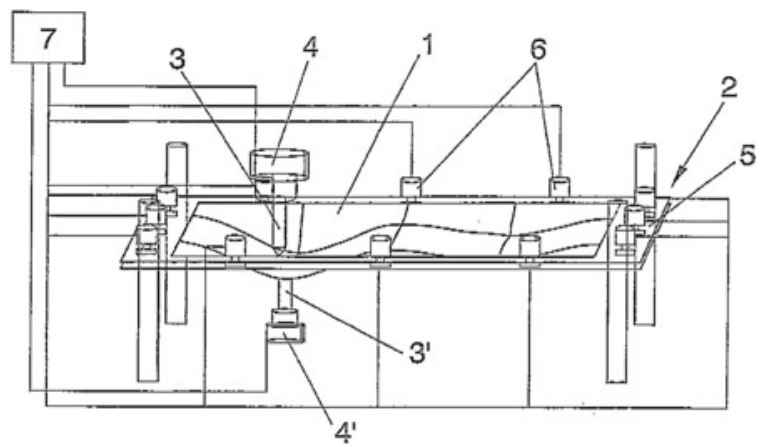

Fig. 23 Patented ISF solution of Rodriguez Gutierrez et al. [19] with 1 sheet, 2 fastening, 3 first tool, 3': second tool, 4 mounting head of first tool, 4': mounting head of second tool, 5 peripheral frame, 6 piezoelectric actuators, 7 controller
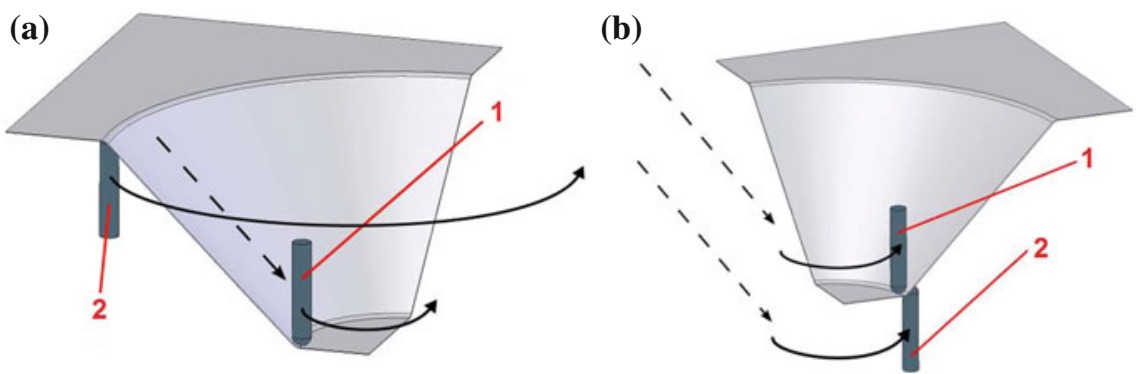

Fig. 24 a Forming strategy "A", b Forming strategy "B" where 1 first forming tool, 2 second forming tool

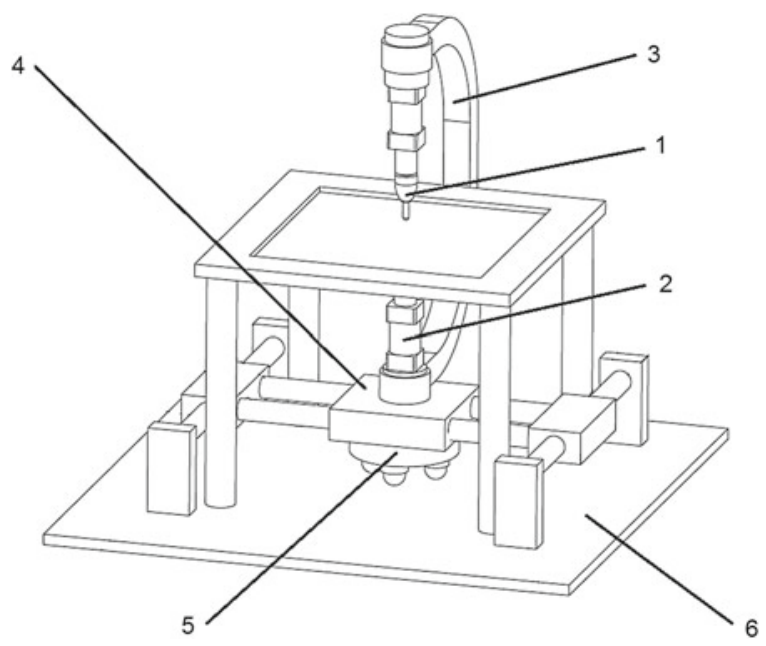

Fig. 25 Patented ISF solution of Somlo and Paniti [22] where 1 first forming tool, 2 second forming tool with linear actuator, $3 \mathrm{C}$-frame, 4 linear slide system, 5 ball transfer unit, 6 base plate 


\section{Novel TPIF Solutions with Counter Tool}

In the previous years an enhancement in ISF could be observed which manifested itself in a number of R\&D projects and patents [8] all over the world.

SPIF has several limitations; for example it needs a backing plate, and forming of convex and concave parts on the same sheet is only feasible if the clamping frame is released.

The patented solution of Rodriguez Gutierrez et al. [19] overcame these issues by the synchronised movement of two forming tools (see Fig. 23). The synchronisation of the two tools is solved by a common controller.

Forming experiments on the prototype of the previously mentioned patent showed that the set-up allows two main forming strategies (see Fig. 24) for the manufacturing of sheet metal parts and the local support of the second tool gives better results in terms of formability [23]. Experiments were carried out on a $0.6 \mathrm{~mm}$ thick aluminium sheet (Al 1050).

In the case of strategy "A" the second tool acts as a backing plate, moving with the first tool, but does not leave the starting contour of the supporting tool path. In the case of strategy "B" the second tool moves with the forming tool, ensuring continuous support. The same solution with two synchronised industrial robots was used by Meier et al. [13]. They also observed better results with strategy "B"; their names for the abovementioned strategies were "duplex incremental forming with peripheral support" (DPIF-P) and "duplex incremental forming with local support" (DPIF-L) respectively.

Meier et al. [13] extended the analytical model of Silva et al. [20] for rotational symmetric SPIF by the superimposed pressure induced by the second (supporting) tool to analyse the influence of the parameters in DPIF-L.

Similar results can be reached with the patented solution of Somlo and Paniti [22]. The device also makes it possible to form a sheet from both sides, but instead of the synchronisation of a minimum $2 \times 3$ axis the $x-y$ motion of the second forming tool is copied with a C-frame (see a variant of this in Fig. 25).

In Fig. 25 a variant of the patented device is illustrated with a linear slide system which ensures a smooth motion in the $x-y$ direction. The axes of the linear slide system are secured against deflection by a ball transfer unit containing a minimum of one iron ball. The ball transfer unit is placed on a smooth base plate.

This device can be used as an external axis in some milling machine centres and in robotic cells. A detailed description of the solution can be found in [22].

\section{Conclusions}

In this study we intended to show the importance of an adequate sheet thickness measurement solution due to the limitations of thickness prediction approaches.

The presented results showed that the Hall sensor based thickness measurement method for SPIF is capable of measuring the thickness in the small plastic zone of the 
sheet and can detect stabilised thickness areas. The results verified that the solution can be coupled with the adaptive control algorithm presented in [16]. Experiments showed that in the examined cases the monitoring of the pre-defined critical value was able to indicate the need for a tool path change. Fracture occurred only after the execution of the tool path with smaller step depth. Furthermore some novel solutions were introduced in TPIF / DPIF (duplex incremental forming).

Acknowledgments The described work was carried out as part of the TÁMOP-4.2.2/B-10/1-20100008 project in the framework of the New Hungarian Development Plan. The realization of this project is supported by the European Union, co-financed by the European Social Fund. The author would like to also thank the Institute for Computer Science and Control, Hungarian Academy of Sciences, for its support.

\section{References}

1. AD22151 type Hall sensor IC. http://www.analog.com/static/imported-files/data_sheets/ AD22151.pdf

2. Aerens, R., Eyckens, P., Van Bael, A., Duflou, J.: Force prediction for single point incremental forming deduced from experimental and FEM observations. Int. J. Adv. Manuf. Technol. 46, 969-982 (2010)

3. Ambrogio, G., Filice, L., Micari, F.: A force measuring based strategy for failure prevention in incremental forming. J. Mater. Process. Technol. 177(1-3), 413-416 (2006)

4. Attanasio, A., Ceretti, E., Giardini, C.: Optimization of tool path in two points incremental forming. J. Mater. Process. Technol. 177(1-3), 409-412 (2006)

5. Avitzur, B., Yang, C.T.: Analysis of power spinning of cones, J. Eng. Ind.-Trans. ASME, B 82, 231-245 (1960)

6. Bambach, M.: A geometrical model of the kinematics of incremental sheet forming for the prediction of membrane strains and sheet thickness. J. Mater. Process. Technol. 210(12), 1562$1573(2010)$

7. Dejardin, S., Gelin, J.-C., Thibaud, S.: On-line thickness measurement in incremental sheet forming process. Proceedings of the 13th international conference on metal forming, Toyohashi, 19-22 Sept 2010, pp. 938-941

8. Emmens, W.C., Sebastiani, G., van den Boogaard, A.H.: The technology of incremental sheet forming — a brief review of the history. J. Mater. Process. Technol. 210(8), 981-997 (2010). doi:10.1016/j.jmatprotec.2010.02.014

9. He, S., Gu, J., Sol, H., Van Bael, A., van Houtte, P., Tunckol, Y., Duflou, J.R.: Determination of strain in incremental sheet forming process. Key Eng. Mater. 344, 503-510 (2007). doi:10. 4028/www.scientific.net/KEM.344.503

10. Hirt, G., Ames, J., Bambach, M., Kopp, R.: Modeling and experimental evaluation of the incremental CNC sheet metal forming process. CIRP Ann. Manuf. Technol. 53(1), 203-206 (2004)

11. Hussain, G., Gao, L.: A novel method to test the thinning limit of sheet metal in negative incremental forming. Int. J. Mach. Tool. Manuf. 47, 419-435 (2007)

12. Leszak, E.: Apparatus and process for incremental dieless forming, US Patent 3342051A1 (1967)

13. Meier, H., Magnus, C., Smukala, V.: Impact of superimposed pressure on dieless incremental sheet metal forming with two moving tools. CIRP Ann. Manuf. Technol. 60(1), 327-330 (2011)

14. Datasheet of the MOTENC-Lite PCI card. http://www.vitalsystem.com/web/motion/ motionLite.php 
15. Paniti, I., Paroczi, A.: Design and modeling of integrated Hall-effect sensor based online thickness measurement device for incremental sheet forming processes. In: Proceedings of the 2011 IEEE/ASME International Conference on Advanced Intelligent Mechatronics (AIM), http://ieeexplore.ieee.org/xpl/articleDetails.jsp?arnumber=6027146pp. 297-302. Budapest, Hungary, (2011)

16. Paniti, I., Rauschecker, U.: Integration of incremental sheet forming with an adaptive control into cloud manufacturing. In: Proceedings of the 14th International Conference on Modern Information Technology in the Innovation Processes of Industrial Enterprises, MITIP2012, pp. 119-128. Budapest, 24-26 Oct (2012)

17. Powell, N., Andrew, C.: Incremental forming of flanged sheet metal components without dedicated dies. IMECHE part B. J. Eng. Manuf. 206, 41-47 (1992)

18. Rauch, M., Hascoet, J.-Y., Hamann, J.-C., Plenel, Y.: Tool path programming optimization for incremental sheet forming applications. CAD Comput. Aided Des. 41(12), 877-885 (2009)

19. Rodriguez Gutierrez, P.P., Rivero Rastrero, M.A., Maidagan Onandia, E.: Machine for shaping sheet metal and shaping method, EU Patent EP1977842 (2006)

20. Silva, M.B., Skjoedt, M., Martins, P.A.F., Bay, N.: Single-point incremental forming and formability-failure diagrams. J. Strain Anal. Eng. Des. 43(1), 15-36 (2008)

21. Silva, M.B., Skjoedt, M., Bay, N., Martins, P.A.F.: Revisiting single-point incremental forming and formability/failure diagrams by means of finite elements and experimentation. J. Strain Anal. Eng. Des. 44, 221-234 (2009). doi:10.1243/03093247JSA522

22. Somlo, J., Paniti, I.: Device for two sided incremental sheet forming. EU Patent EP2505279 (2012)

23. Tisza, M., Paniti, I., Kovács, P.Z.: Experimental and numerical study of a milling machine-based dieless incremental sheet forming. Int. J. Mater. Form. 3(1), 441-446 (2010)

24. Van Bael, A., He, S., Van Houtte, P., Tunkol, Y., Verbert, J., Duflou, J.R.: Study on the thinning during single point incremental forming of aluminium sheets. Presented at the 24th international deep-drawing research group congress, p. 12. Besançon, France (2005) http://www.ens2m.fr/ iddrg 2005

25. Young, D., Jeswiet, J.: Wall thickness variations in single-point incremental forming. J. Eng. Manuf. Part B 218, 1453-1459 (2004) 